\title{
ASYMPTOTICS FOR ORTHOGONAL POLYNOMIALS OFF THE CIRCLE
}

\author{
R. KHALDI AND R. BENZINE
}

Received 29 April 2003 and in revised form 30 September 2003

We study the strong asymptotics of orthogonal polynomials with respect to a measure of the type $d \mu / 2 \pi+\sum_{j=1}^{\infty} A_{j} \delta\left(z-z_{k}\right)$, where $\mu$ is a positive measure on the unit circle $\Gamma$ satisfying the Szegö condition and $\left\{z_{j}\right\}_{j=1}^{\infty}$ are fixed points outside $\Gamma$. The masses $\left\{A_{j}\right\}_{j=1}^{\infty}$ are positive numbers such that $\sum_{j=1}^{\infty} A_{j}<+\infty$. Our main result is the explicit strong asymptotic formulas for the corresponding orthogonal polynomials.

\section{Introduction}

Let $\nu$ be a finite positive measure defined on the Borelian $\sigma$-algebra of $\mathbb{C}$ and concentrated on the set $\Gamma \cup\left\{z_{k}\right\}_{k=1}^{\infty}$, where $\Gamma=\{z \in \mathbb{C}:|z|=1\}$ and $z_{k}$ are such that $\left|z_{k}\right|>1$. The measure $v$ is defined as follows:

$$
\nu=\frac{\mu}{2 \pi}+\sum_{k=1}^{\infty} A_{k} \delta\left(z-z_{k}\right),
$$

where $\mu$ is concentrated on $\Gamma$ and is absolutely continuous with respect to the Lebesgue measure $d \theta$ on $[-\pi,+\pi]$, that is,

$$
d \mu(\theta)=\rho(\theta) d \theta, \quad \rho \geq 0, \rho \in L^{1}([-\pi,+\pi], d \theta) .
$$

The masses $\left\{A_{k}\right\}_{k=1}^{\infty}$ satisfy

$$
A_{k}>0, \quad \sum_{k=1}^{\infty} A_{k}<+\infty,
$$

and $\delta\left(z-z_{k}\right)$ is the Dirac measure supported at the point $z_{k}$.

We denote by $\left\{\Phi_{n}(z)\right\}$ and $\left\{\varphi_{n}(z)\right\}$ the systems of orthonormal polynomials associated to the measures $\nu$ and $\mu / 2 \pi$, respectively.

For $\ell \geq 1$, we denote $\nu_{\ell}=\mu / 2 \pi+\sum_{k=1}^{\ell} A_{k} \delta\left(z-z_{k}\right)$, and by $\left\{\Phi_{n}^{\ell}(z)\right\}$ we denote the system of orthonormal polynomials associated to $\nu_{\ell}$. 
$\left\{\Phi_{n}\right\}_{n \in \mathbb{N}}$ satisfy the following orthonormality relations:

$$
\begin{gathered}
\Phi_{n}(z)=\gamma_{n} z^{n}+\cdots \quad\left(\gamma_{n}>0\right) \\
\frac{1}{2 \pi} \int_{-\pi}^{+\pi} \Phi_{m}(z) \overline{\Phi_{n}(z)} \rho(\theta) d \theta+\sum_{k=1}^{\infty} A_{k} \Phi_{m}\left(z_{k}\right) \overline{\Phi_{n}\left(z_{k}\right)}=\delta_{m, n} \\
m, n=0,1,2, \ldots, z=e^{i \theta}
\end{gathered}
$$

For the system $\left\{\varphi_{n}\right\}_{n \in \mathbb{N}}$, one gets

$$
\begin{gathered}
\varphi_{n}(z)=k_{n} z^{n}+\cdots \quad\left(k_{n}>0\right), \\
\frac{1}{2 \pi} \int_{-\pi}^{+\pi} \varphi_{m}(z) \overline{\varphi_{n}(z)} \rho(\theta) d \theta=\delta_{m, n}, \quad m, n=0,1,2, \ldots, z=e^{i \theta} .
\end{gathered}
$$

Finally we also have the system $\left\{\Phi_{n}^{\ell}\right\}_{n \in \mathbb{N}}$ :

$$
\begin{gathered}
\Phi_{n}^{\ell}(z)=\gamma_{n}^{\ell} z^{n}+\cdots \quad\left(\gamma_{n}^{\ell}>0\right) \\
\frac{1}{2 \pi} \int_{-\pi}^{+\pi} \Phi_{m}^{\ell}(z) \overline{\Phi_{n}^{\ell}(z)} \rho(\theta) d \theta+\sum_{k=1}^{\ell} A_{k} \Phi_{m}^{\ell}\left(z_{k}\right) \overline{\Phi_{n}^{\ell}\left(z_{k}\right)}=\delta_{m, n} \\
m, n=0,1,2, \ldots, z=e^{i \theta} .
\end{gathered}
$$

The goal of this paper is the study of the asymptotic behavior of the polynomials $\Phi_{n}(z)$ outside $\Gamma$.

A similar study has been done by Benzine in [2] and Khaldi and Benzine in [6], in the case of a curve instead of a circle. To prove their results, Benzine and Khaldi imposed some conditions on the measure $v$ which are difficult to check. In $[2,6] v$ is concentrated on $E \cup\left\{z_{k}\right\}_{k=1}^{\infty}$, E being a curve.

In the present work, we prove the same result in the case of a circle instead of a curve with some assumptions on $v$ which are not difficult to check.

To get the asymptotic formula of $\Phi_{n}(z)$ (Theorem 5.4), we prove two intermediate results, Theorems 5.1 and 5.2. Theorem 5.1 establishes that the coefficients $\gamma_{n}^{\ell}$ of $z^{n}$ of the polynomials $\Phi_{n}^{\ell}(z)$ tend (when $\ell \rightarrow \infty$ ) to the coefficient $\gamma_{n}$ of $z^{n}$ of the polynomials $\Phi_{n}(z)$.

The new conditions on the measure $\nu$, Theorems 5.1 and 5.2, as well as their consequences on the proof of Theorem 5.3 constitute our main contribution with respect to previous works by Benzine and Khaldi $[2,6]$.

The asymptotic behavior of the orthogonal polynomials $\left\{\varphi_{n}(z)\right\}$ has been deeply studied by Szegö [14] and Smirnov $[11,12,13]$, when $\mu$ is absolutely continuous, and by Akhiezer and Krein [1] and Geronimus [4] if $\mu$ is not absolutely continuous.

The asymptotic behavior of the polynomials $\left\{\Phi_{n}^{\ell}(z)\right\}$ has been established by Li and Pan [9] in the case where the measure $\mu$ is not absolutely continuous, and by Kaliaguine and Benzine [5] in the case of a curve with $\mu$ an absolutely continuous measure. 


\section{The space $H^{2}(G, \rho)$}

We denote $G=\{z \in \mathbb{C}:|z|>1\} \cup\{\infty\}$. We say that $f \in H^{2}(G)$ if $f$ is analytic in $G$ and $\int_{C_{r}}|f(z)|^{2}|d z| \leq C, r>1, C_{r}=\{z \in \mathbb{C}:|z|=r\}$, and $C$ is a constant independent of $r$.

In what follows, we suppose that the weight function $\rho$ (which defines the measure $\mu$ ) belongs to the Szegö class, that is,

$$
\rho \geq 0, \quad \rho \in L^{1}([-\pi,+\pi], d \theta), \quad \log (\rho) \in L^{1}([-\pi,+\pi], d \theta) .
$$

This allows us to construct the so-called Szegö function $D$ associated with the domain $G$ and weight function $\rho$ :

$$
D(w)=\exp \left\{\frac{1}{4 \pi} \int_{-\pi}^{+\pi} \log (\rho(t)) \frac{w+e^{-i t}}{w-e^{-i t}} d t\right\} \quad(|w|>1)
$$

such that

$$
\begin{gathered}
D \in H^{2}(G), \quad D(z) \neq 0, \quad D(\infty)>0, \quad\left|\tilde{D}\left(e^{i \theta}\right)\right|^{2}=\rho(\theta), \\
(\text { a.e. on }[-\pi,+\pi]),
\end{gathered}
$$

where $\tilde{D}$ is the angular limit of $D$. We say that $f \in H^{2}(G, \rho)$ if and only if $f(z)$ is analytic in $G$ and $(f \cdot D) \in H^{2}(G)$. Finally, the space $L^{2}(\Gamma, \rho|d \xi|)$ is the space of functions $f$ defined on the unit circle $\Gamma$, with values in $\mathbb{C}$ and for which $\int_{-\pi}^{+\pi}\left|f\left(e^{i \theta}\right)\right|^{2} \rho(\theta) d \theta<+\infty$. Let $f$ and $g$ be in $L^{2}(\Gamma, \rho|d \xi|)$, we define

$$
\begin{gathered}
\langle f, g\rangle_{L^{2}(\Gamma, \rho|d \xi|)}=\frac{1}{2 \pi} \int_{-\pi}^{+\pi} f\left(e^{i \theta}\right) \overline{g\left(e^{i \theta}\right)} \rho(\theta) d \theta, \\
\|f\|_{L^{2}(\Gamma, \rho|d \xi|)}^{2}=\langle f, f\rangle_{L^{2}(\Gamma, \rho|d \xi|)},
\end{gathered}
$$

then $\left(L^{2}(\Gamma, \rho|d \xi|),\|\cdot\|_{L^{2}(\Gamma, \rho|d \xi|)}\right)$ is a Hilbert space. We summarize the basic properties of the space $H^{2}(G, \rho)$ in the following theorem and lemma.

Theorem 2.1 [5]. Let $f \in H^{2}(G, \rho)$. Then $f$ has a.e. in $\Gamma$ an angular limit which is denoted by $\tilde{f}, \tilde{f}\left(e^{i \theta}\right)=\lim _{z \rightarrow e^{i \theta}} f(z)$. Moreover,

(1) $\tilde{f} \in L^{2}(\Gamma, \rho|d \xi|)$,

(2) $\left(H^{2}(G, \rho),\|\cdot\|_{\rho}\right)$ is a Hilbert space, where

$$
\begin{aligned}
\|f\|_{\rho}^{2} & =\langle f, f\rangle_{\rho}, \\
\langle f, g\rangle_{\rho}=\langle\tilde{f}, \tilde{g}\rangle_{L^{2}(\Gamma, \rho|d \xi|)} & =\frac{1}{2 \pi} \int_{-\pi}^{+\pi} \tilde{f}\left(e^{i \theta}\right) \overline{\tilde{g}\left(e^{i \theta}\right)} \rho(\theta) d \theta
\end{aligned}
$$

for $f \in H^{2}(G, \rho)$ and $g \in H^{2}(G, \rho)$,

(3) if $f \in H^{2}(G, \rho)$, then for every compact set $K \subset G$, there is a constant $C(K)(C(K)$ depends only on $K$ ) such that

$$
\sup _{z \in K}|f(z)| \leq C(K)\|f\|_{\rho} .
$$


Lemma 2.2 [5, page 35]. Let $\left\{f_{n}\right\}$ be a sequence of functions from $H^{2}(G, \rho)$ and

(i) $f_{n} \rightarrow f$ uniformly on the compact sets of $G$,

(ii) $\left\|f_{n}\right\|_{\rho} \leq M$ (constant).

Then $f \in H^{2}(G, \rho)$ and $\|f\|_{\rho} \leq \liminf \left\|f_{n}\right\|_{\rho}$.

\section{The set of measures $A$}

3.1. Definitions. We will give in this part the new conditions on the discrete part of the measure $v$ which allows us later to get the asymptotic formula of the orthonormal polynomials $\left\{\Phi_{n}(z)\right\}$.

We need to introduce some notation. The $*$-transform $\left(P_{n}\right)^{*}(z)$ of a polynomial $P_{n}(z)$ of degree $n$ is defined as

$$
\left(P_{n}\right)^{*}(z)=\overline{z^{n} P_{n}\left(\frac{1}{\bar{z}}\right)}
$$

Definition 3.1. Say that the measure $\nu=\mu / 2 \pi+\sum_{k=1}^{\infty} A_{k} \delta\left(z-z_{k}\right)$ belongs to the class $A$ (and $\nu \in A$ ) if the absolutely continuous part $\mu / 2 \pi$ satisfies the Szegö condition and if the discrete part $\sum_{k=1}^{\infty} A_{k} \delta\left(z-z_{k}\right)$ of $\nu$ satisfies

$$
\begin{gathered}
\sum_{k=1}^{\infty}\left(\left|z_{k}\right|-1\right)<+\infty, \\
\frac{\left|z_{\ell+1}\right|^{2}-1}{A_{\ell+1}} \geq\left|\left(\Phi_{n}^{\ell}\right)^{*}\left(z_{\ell+1}\right)\right|^{2} \text { for } l=0,1,2, \ldots,
\end{gathered}
$$

where

$$
\left(\Phi_{n}^{0}\right)^{*}(z)=\left(\varphi_{n}\right)^{*}(z)
$$

Condition (3.2) guarantees the convergence of the Blaschke product $B_{\infty}(z)$ associated to the points $\left\{z_{k}\right\}_{k=1}^{\infty}$ :

$$
B_{\infty}(z)=\prod_{k=1}^{\infty} \frac{z-z_{k}}{z \overline{z_{k}}-1} \frac{\left|z_{k}\right|^{2}}{z_{k}} .
$$

As an example of families of points and masses satisfying condition (3.3), we can take the subsequences of the points $\left\{z_{k}\right\}_{k=1}^{\infty}$ and the masses $\left\{A_{k}\right\}_{k=1}^{\infty}$ of the following form:

$$
\left\{z_{k} \in \mathbb{C}:\left|z_{k}\right|=1+\frac{1}{k^{2}}\right\}_{k=1}^{\infty}, \quad\left\{A_{k}=\frac{1}{2^{k}}\right\}_{k=1}^{\infty} .
$$

Such a choice is possible since $\lim _{k \rightarrow \infty}\left(\left(\left|z_{k}\right|^{2}-1\right) / A_{k}\right)=+\infty$ and the sequence $\left\{\left(\Phi_{n}^{0}\right)^{*}(z)\right\}_{n=1}^{\infty}$ is uniformly bounded in $|z|<1 / \rho$ for

$$
\rho=\limsup \left|\frac{\varphi_{n}(0)}{k_{n}}\right|^{1 / n}<1 .
$$


On the other hand, the sequence $\left\{\left(\Phi_{n}^{\ell}\right) *(z)\right\}_{n=1}^{\infty}(\ell \geq 1)$ is locally bounded in $|z|<1 / \tau<$ $1 / \rho$, where

$$
\tau=\max _{1 \leq j \leq \ell}\left\{\left|z_{j}\right|^{-1}\right\}=\left|z_{\ell}\right|^{-1}
$$

(see [9, pages 66-67]).

\section{Extremal properties of the orthogonal polynomials}

In this section and the following one, we assume that the measure $\nu$ belongs to the class $A$. We denote by $\mathscr{P}_{n}$ the vector space of all polynomials of degree at most $n$, and by $\mathscr{P}_{n 1}$ the set of monic polynomials of degree exactly equal to $n$. It is easy to see that the polynomial $\left(1 / \gamma_{n}\right) \Phi_{n}$ is the optimal solution of the following extremal problem:

$$
\left(\frac{1}{\gamma_{n}}\right)^{2}=\left\|\frac{1}{\gamma_{n}} \Phi_{n}\right\|_{\nu}^{2}=\min \left\{\left\|Q_{n}\right\|_{\nu}^{2}: Q_{n} \in \mathscr{P}_{n 1}\right\}=m_{n}(\nu)
$$

with

$$
\left\|Q_{n}\right\|_{\nu}^{2}=\frac{1}{2 \pi} \int_{-\pi}^{+\pi}\left|Q_{n}\left(e^{i \theta}\right)\right|^{2} \rho(\theta) d \theta+\sum_{k=1}^{\infty} A_{k}\left|Q_{n}\left(z_{k}\right)\right|^{2} .
$$

We define $\mu_{n}(\nu), \mu_{n}(\ell), \mu_{n}(\rho), \mu(\rho), \mu(\nu), m_{n}(\rho)$, and $m_{n}(\ell)$ to be the optimal values of the extremal problems

$$
\begin{aligned}
& \mu_{n}(\nu)=\min \left\{\frac{1}{2 \pi} \int_{-\pi}^{+\pi}\left|\psi_{n}\left(e^{i \theta}\right)\right|^{2} \rho(\theta) d \theta\right. \\
& \left.\quad+\sum_{k=1}^{\infty} A_{k}\left|z_{k}\right|^{2 n}\left|\psi_{n}\left(z_{k}\right)\right|^{2}: \psi_{n}=\frac{Q_{n}}{z^{n}}, Q_{n} \in \mathscr{P}_{n}, \psi_{n}(\infty)=1\right\}, \\
& \mu_{n}(\ell)=\min \left\{\frac{1}{2 \pi} \int_{-\pi}^{+\pi}\left|\psi_{n}\left(e^{i \theta}\right)\right|^{2} \rho(\theta) d \theta\right. \\
& \left.\quad+\sum_{k=1}^{\ell} A_{k}\left|z_{k}\right|^{2 n}\left|\psi_{n}\left(z_{k}\right)\right|^{2}: \psi_{n}=\frac{Q_{n}}{z^{n}}, Q_{n} \in \mathscr{P}_{n}, \psi_{n}(\infty)=1\right\}, \\
& \mu_{n}(\rho)=\min \left\{\frac{1}{2 \pi} \int_{-\pi}^{+\pi}\left|\psi_{n}\left(e^{i \theta}\right)\right|^{2} \rho(\theta) d \theta: \psi_{n}=\frac{Q_{n}}{z^{n}},\right. \\
& \left.Q_{n} \in \mathscr{P}_{n}, \psi_{n}(\infty)=1\right\},
\end{aligned}
$$




$$
\begin{gathered}
\mu(\rho)=\inf \left\{\frac{1}{2 \pi} \int_{-\pi}^{+\pi}\left|\psi\left(e^{i \theta}\right)\right|^{2} \rho(\theta) d \theta: \psi \in H^{2}(G, \rho), \psi(\infty)=1\right\}, \\
\mu(\nu)=\inf \left\{\frac{1}{2 \pi} \int_{-\pi}^{+\pi}\left|\psi\left(e^{i \theta}\right)\right|^{2} \rho(\theta) d \theta: \psi \in H^{2}(G, \rho), \psi(\infty)=1,\right. \\
\left.\psi\left(z_{k}\right)=0, k=1,2, \ldots\right\}, \\
m_{n}(\ell)=\left(\frac{1}{\gamma_{n}^{\ell}}\right)^{2}=\left\|\frac{1}{\gamma_{n}^{\ell}} \Phi_{n}^{\ell}\right\|_{\gamma_{\ell}}^{2}=\min \left\{\left\|Q_{n}\right\|_{\nu_{\ell}}^{2}: Q_{n} \in \mathscr{P}_{n 1}\right\}
\end{gathered}
$$

with

$$
\begin{gathered}
\left\|Q_{n}\right\|_{\nu_{\ell}}^{2}=\frac{1}{2 \pi} \int_{-\pi}^{+\pi}\left|Q_{n}\left(e^{i \theta}\right)\right|^{2} \rho(\theta) d \theta+\sum_{k=1}^{\ell} A_{k}\left|Q_{n}\left(z_{k}\right)\right|^{2} \\
m_{n}(\rho)=\left(\frac{1}{k_{n}}\right)^{2}=\left\|\frac{1}{k_{n}} \varphi_{n}\right\|_{\rho}^{2}=\min \left\{\left\|Q_{n}\right\|_{\rho}^{2}: Q_{n} \in \mathscr{P}_{n 1}\right\} .
\end{gathered}
$$

Denote by $\psi_{n}^{\infty}, \psi_{n}^{\ell}, \psi_{n}^{\rho}, \hat{\psi}$, and $\psi^{\infty}$ the optimal solutions of the extremal problems (4.3), (4.4), (4.5), (4.6), and (4.7), respectively. We are now ready to state the lemmas which we need in the sequel.

The proof of Lemma 4.1 is trivial.

Lemma 4.1. The optimal solutions $\psi_{n}^{\infty}, \psi_{n}^{\ell}, \psi_{n}^{\rho}, \hat{\psi}$, and $\psi^{\infty}$, and the extremal values $\mu_{n}(\nu)$, $m_{n}(\nu), \mu_{n}(\ell), m_{n}(\ell), \mu_{n}(\rho)$, and $m_{n}(\rho)$ verify

$$
\begin{aligned}
& \psi_{n}^{\infty}(z)=\frac{1}{\gamma_{n}} \frac{\Phi_{n}(z)}{z^{n}}, \quad \psi_{n}^{\ell}(z)=\frac{1}{\gamma_{n}^{\ell}} \frac{\Phi_{n}^{\ell}(z)}{z^{n}}, \quad \psi_{n}^{\rho}(z)=\frac{1}{k_{n}} \frac{\varphi_{n}(z)}{z^{n}}, \\
& \mu_{n}(\nu)=m_{n}(\nu), \quad \mu_{n}(\ell)=m_{n}(\ell), \quad \mu_{n}(\rho)=m_{n}(\rho) .
\end{aligned}
$$

Lemma 4.2 [2]. Let $\varphi \in H^{2}(G, \rho)$ be such that $\varphi(\infty)=1$ and $\varphi\left(z_{k}\right)=0, k=1,2, \ldots$, and $B_{\infty}$ the Blaschke product (3.5). Then

$$
B_{\infty} \in H^{2}(G, \rho), \quad B_{\infty}(\infty)=1, \quad\left|\widetilde{B}_{\infty}\left(e^{i \theta}\right)\right|=\prod_{k=1}^{\infty}\left|z_{k}\right|
$$

with

$$
\begin{aligned}
\widetilde{B}_{\infty}\left(e^{i \theta}\right) & =\lim _{z \rightarrow e^{i \theta}} B_{\infty}(z), \\
\frac{\varphi}{B_{\infty}} & \in H^{2}(G, \rho) .
\end{aligned}
$$


Lemma 4.3. The optimal value $\mu(\nu)$ verifies the following equality:

$$
\mu(\nu)=\mu\left(\rho_{1}\right) \quad \text { with } \rho_{1}=\rho \cdot\left(\prod_{k=1}^{\infty}\left|z_{k}\right|\right)^{2}
$$

Proof. Let $\varphi \in H^{2}(G, \rho)$ be such that $\varphi(\infty)=1$ and verifies

$$
\begin{aligned}
\mu\left(\rho_{1}\right) & =\frac{1}{2 \pi} \int_{-\pi}^{+\pi}\left|\varphi\left(e^{i \theta}\right)\right|^{2} \rho_{1}(\theta) d \theta \\
& =\frac{1}{2 \pi} \int_{-\pi}^{+\pi}\left|\varphi\left(e^{i \theta}\right)\right|^{2}\left|\widetilde{B}_{\infty}\left(e^{i \theta}\right)\right|^{2} \rho(\theta) d \theta .
\end{aligned}
$$

Now consider the function $\psi=\varphi \cdot B_{\infty}$. Note that

$$
\psi \in H^{2}(G, \rho), \quad \psi(\infty)=1, \quad \psi\left(z_{k}\right)=0, \quad k=1,2, \ldots
$$

Then, considering (4.7),

$$
\begin{aligned}
\mu\left(\rho_{1}\right) & =\frac{1}{2 \pi} \int_{-\pi}^{+\pi}\left|\varphi\left(e^{i \theta}\right)\right|^{2} \rho_{1}(\theta) d \theta \\
& =\frac{1}{2 \pi} \int_{-\pi}^{+\pi}\left|\psi\left(e^{i \theta}\right)\right|^{2} \rho(\theta) d \theta \geq \mu(\nu) .
\end{aligned}
$$

Conversely, consider $\psi_{1} \in H^{2}(G, \rho)$ such that

$$
\begin{gathered}
\psi_{1}(\infty)=1, \quad \psi_{1}\left(z_{k}\right)=0, \quad k=1,2, \ldots, \\
\mu(\nu)=\frac{1}{2 \pi} \int_{-\pi}^{+\pi}\left|\psi_{1}\left(e^{i \theta}\right)\right|^{2} \rho(\theta) d \theta .
\end{gathered}
$$

Consider the function

$$
\varphi_{1}(z)=\frac{\psi_{1}(z)}{B_{\infty}(z)}
$$

Lemma 4.2 implies that $\varphi_{1} \in H^{2}(G, \rho)$ and $\varphi_{1}(\infty)=1$. Then

$$
\begin{aligned}
\mu(\nu) & =\frac{1}{2 \pi} \int_{-\pi}^{+\pi}\left|\psi_{1}\left(e^{i \theta}\right)\right|^{2} \rho(\theta) d \theta \\
& =\frac{1}{2 \pi} \int_{-\pi}^{+\pi}\left|\varphi_{1}\left(e^{i \theta}\right)\right|^{2}\left|\widetilde{B}_{\infty}\left(e^{i \theta}\right)\right|^{2} \rho(\theta) d \theta \\
& =\frac{1}{2 \pi} \int_{-\pi}^{+\pi}\left|\varphi_{1}\left(e^{i \theta}\right)\right|^{2} \rho_{1}(\theta) d \theta \geq \mu\left(\rho_{1}\right) .
\end{aligned}
$$


44 Asymptotics for orthogonal polynomials off the circle

Lemma $4.4[2]$. For $\mu(\nu)$,

$$
\begin{gathered}
\mu(\nu)=\mu(\rho) \cdot\left[\prod_{k=1}^{\infty}\left|z_{k}\right|\right]^{2}, \\
\psi^{\infty}(z)=\hat{\psi}(z) \cdot B_{\infty}(z),
\end{gathered}
$$

where $\hat{\psi}$ and $\psi^{\infty}$ are, respectively, the optimal solutions of the extremal problems (4.6) and (4.7).

\section{Main results}

Theorem 5.1. Let $\nu=\mu / 2 \pi+\sum_{k=1}^{\infty} A_{k} \delta\left(z-z_{k}\right)$, where $\mu$ is a positive measure whose support is the unit circle,

$$
\begin{gathered}
\sum_{k=1}^{\infty} A_{k}<+\infty, \quad A_{k}>0 \\
\sum_{k=1}^{\infty}\left(\left|z_{k}\right|-1\right)<+\infty, \quad\left|z_{k}\right|>1 .
\end{gathered}
$$

Then

$$
\begin{aligned}
\lim _{\ell \rightarrow \infty} \mu_{n}(\ell) & =\mu_{n}(\nu), \\
\lim _{\ell \rightarrow \infty} \gamma_{n}^{\ell} & =\gamma_{n} .
\end{aligned}
$$

Proof. The extremal property of $\left(1 / \gamma_{n}^{\ell}\right) \Phi_{n}^{\ell}(z)$ yields

$$
\begin{aligned}
\mu_{n}(\nu)= & \left(\frac{1}{\gamma_{n}}\right)^{2}=\frac{1}{2 \pi} \int_{-\pi}^{+\pi}\left|\frac{1}{\gamma_{n}} \Phi_{n}\left(e^{i \theta}\right)\right|^{2} \rho(\theta) d \theta \\
& +\sum_{k=1}^{\infty} A_{k}\left|\frac{1}{\gamma_{n}} \Phi_{n}\left(z_{k}\right)\right|^{2} \\
\geq & \frac{1}{2 \pi} \int_{-\pi}^{+\pi}\left|\frac{1}{\gamma_{n}} \Phi_{n}\left(e^{i \theta}\right)\right|^{2} \rho(\theta) d \theta+\sum_{k=1}^{\ell} A_{k}\left|\frac{1}{\gamma_{n}} \Phi_{n}\left(z_{k}\right)\right|^{2} \\
\geq & \frac{1}{2 \pi} \int_{-\pi}^{+\pi}\left|\frac{1}{\gamma_{n}^{\ell}} \Phi_{n}^{\ell}\left(e^{i \theta}\right)\right|^{2} \rho(\theta) d \theta+\sum_{k=1}^{\ell} A_{k}\left|\frac{1}{\gamma_{n}^{\ell}} \Phi_{n}^{\ell}\left(z_{k}\right)\right|^{2} \\
= & \left(\frac{1}{\gamma_{n}^{\ell}}\right)^{2}=\mu_{n}(\ell) .
\end{aligned}
$$

Then

$$
\frac{\mu_{n}(\nu)}{\mu_{n}(\ell)} \geq 1 \quad \forall \ell>0 .
$$


On the other hand (see (4.3) and (4.4)),

$$
\begin{aligned}
\mu_{n}(\nu) \leq & \frac{1}{2 \pi} \int_{-\pi}^{+\pi}\left|\frac{1}{\gamma_{n}^{\ell}} \Phi_{n}^{\ell}\left(e^{i \theta}\right)\right|^{2} \rho(\theta) d \theta+\sum_{j=1}^{\infty} A_{j}\left|\frac{1}{\gamma_{n}^{\ell}} \Phi_{n}^{\ell}\left(z_{j}\right)\right|^{2} \\
= & \frac{1}{2 \pi} \int_{-\pi}^{+\pi}\left|\frac{1}{\gamma_{n}^{\ell}} \Phi_{n}^{\ell}\left(e^{i \theta}\right)\right|^{2} \rho(\theta) d \theta \\
& +\sum_{j=1}^{\ell} A_{j}\left|\frac{1}{\gamma_{n}^{\ell}} \Phi_{n}^{\ell}\left(z_{j}\right)\right|^{2}+\sum_{j=\ell+1}^{\infty} A_{j}\left|\frac{1}{\gamma_{n}^{\ell}} \Phi_{n}^{\ell}\left(z_{j}\right)\right|^{2} \\
= & \mu_{n}(\ell)+\left(\frac{1}{\gamma_{n}^{\ell}}\right)^{2} \sum_{j=\ell+1}^{\infty} A_{j}\left|\Phi_{n}^{\ell}\left(z_{j}\right)\right|^{2} .
\end{aligned}
$$

The reproducing kernel polynomial $K_{n+1}$ is defined by

$$
K_{n+1}(z, \xi)=\sum_{k=0}^{n} \overline{\varphi_{k}(z)} \varphi_{k}(\xi)
$$

From the reproducing property of $K_{n+1}(\xi, z)$ (see [12]) and the fact that $\Phi_{n}^{\ell}(z) \in \mathscr{P}_{n}$, we get that

$$
\Phi_{n}^{\ell}\left(z_{j}\right)=\frac{1}{2 \pi} \int_{-\pi}^{+\pi} \Phi_{n}^{\ell}\left(e^{i \theta}\right) \overline{K_{n+1}\left(e^{i \theta}, z_{j}\right)} \rho(\theta) d \theta
$$

The Cauchy-Scharwz inequality implies that

$$
\begin{aligned}
& \left|\Phi_{n}^{\ell}\left(z_{j}\right)\right|^{2} \\
& \quad \leq\left(\frac{1}{2 \pi} \int_{-\pi}^{+\pi}\left|\Phi_{n}^{\ell}\left(e^{i \theta}\right)\right|^{2} \rho(\theta) d \theta\right)\left(\frac{1}{2 \pi} \int_{-\pi}^{+\pi}\left|K_{n+1}\left(e^{i \theta}, z_{j}\right)\right|^{2} \rho(\theta) d \theta\right) \\
& \quad \leq \frac{1}{2 \pi} \int_{-\pi}^{+\pi}\left|K_{n+1}\left(e^{i \theta}, z_{j}\right)\right|^{2} \rho(\theta) d \theta=K_{n+1}\left(z_{j}, z_{j}\right) .
\end{aligned}
$$

Equations (5.6) and (5.9) imply that (once we remark that $\left.\mu_{n}(\ell)=1 /\left(\gamma_{n}^{\ell}\right)^{2}\right)$

$$
\begin{aligned}
\mu_{n}(\nu) & \leq \mu_{n}(\ell)\left[1+\sum_{j=\ell+1}^{\infty} A_{j} K_{n+1}\left(z_{j}, z_{j}\right)\right] \\
& \leq \mu_{n}(\ell)\left[1+\sup _{j \geq \ell+1} K_{n+1}\left(z_{j}, z_{j}\right) \sum_{j=\ell+1}^{\infty} A_{j}\right]
\end{aligned}
$$

or else

$$
\frac{\mu_{n}(\nu)}{\mu_{n}(\ell)} \leq 1+\sup _{j \geq \ell+1} K_{n+1}\left(z_{j}, z_{j}\right) \sum_{j=\ell+1}^{+\infty} A_{j} .
$$


46 Asymptotics for orthogonal polynomials off the circle

Using (1.3), (5.5), and (5.11), we obtain

$$
\mu_{n}(\nu) \leq \liminf _{\ell \rightarrow \infty} \mu_{n}(\ell) \leq \limsup _{\ell \rightarrow \infty} \mu_{n}(\ell) \leq \mu_{n}(\nu) \quad \forall n .
$$

This implies that

$$
\lim _{\ell \rightarrow \infty} \mu_{n}(\ell)=\mu_{n}(\nu) \quad \forall n
$$

Relation (5.3) is a direct consequence of (5.13) once we remark that

$$
\mu_{n}(\ell)=\frac{1}{\left(\gamma_{n}^{\ell}\right)^{2}}, \quad \mu_{n}(\nu)=\frac{1}{\left(\gamma_{n}\right)^{2}} .
$$

Theorem 5.2. If $v \in A$, then there holds

$$
\frac{k_{n}}{\gamma_{n}^{\ell}} \leq \prod_{k=1}^{\ell}\left|z_{k}\right|
$$

Proof. We prove (5.15) by induction. We need to introduce some notation. The reproducing kernel polynomial $R_{n}^{l}$ is defined by

$$
R_{n}^{l}(z, \xi)=\sum_{k=0}^{n-1} \overline{\Phi_{k}^{\ell}(z)} \Phi_{k}^{\ell}(\xi) .
$$

In case $\ell=1$, from $[8$, formula 11 , page 61$]$, we have

$$
\left(\frac{k_{n}}{\gamma_{n}^{1}}\right)^{2}=1+A_{1} \frac{k_{n}}{\gamma_{n}^{1}} \Phi_{n}^{1}\left(z_{1}\right) \cdot \overline{\varphi_{n}\left(z_{1}\right)} .
$$

On the other hand,

$$
\Phi_{n}^{1}(z)-\frac{\gamma_{n}^{1}}{k_{n}} \varphi_{n}(z)=-A_{1} \Phi_{n}^{1}\left(z_{1}\right) K_{n}\left(z_{1}, z\right) .
$$

Thus

$$
\frac{k_{n}}{\gamma_{n}^{1}} \Phi_{n}^{1}\left(z_{1}\right)=\frac{\varphi_{n}\left(z_{1}\right)}{1+A_{1} K_{n}\left(z_{1}, z_{1}\right)}
$$

and (5.17) implies that

$$
\left(\frac{k_{n}}{\gamma_{n}^{1}}\right)^{2}=1+\frac{A_{1}\left|\varphi_{n}\left(z_{1}\right)\right|^{2}}{1+A_{1} K_{n}\left(z_{1}, z_{1}\right)} .
$$

Then by using the Christoffel-Darboux formula, we have

$$
\left(\frac{k_{n}}{\gamma_{n}^{1}}\right)^{2}=1+\frac{\left|z_{1}\right|^{2}-1}{\left(\left|z_{1}\right|^{2}-1\right) / A_{1}\left|\varphi_{n}\left(z_{1}\right)\right|^{2}+1-\left|\left(\varphi_{n}\right)^{*}\left(z_{1}\right) / \varphi_{n}\left(z_{1}\right)\right|^{2}}
$$


Taking (3.3) and (3.4), for $l=0$, we get that

$$
\left|\left(\varphi_{n}\right)^{*}\left(z_{1}\right)\right|^{2} \leq \frac{\left|z_{1}\right|^{2}-1}{A_{1}}
$$

Thus, (5.21) and (5.22) imply that

$$
\left(\frac{k_{n}}{\gamma_{n}^{1}}\right)^{2} \leq\left|z_{1}\right|^{2}
$$

We assume the relation (5.15) to be true for the index $\ell$.

Note that the orthonormality of $\Phi_{n}^{\ell}(z)$ yields (on writing $\Phi_{n}^{\ell+1}(z)=\left(\gamma_{n}^{\ell+1} / \gamma_{n}^{\ell}\right) \Phi_{n}^{\ell}(z)+$ $p(z)$, with $\left.p \in \mathscr{P}_{n-1}\right)$

$$
\frac{1}{2 \pi} \int_{-\pi}^{+\pi} \Phi_{n}^{\ell+1}(z) \cdot \overline{\Phi_{n}^{\ell}(z)} \rho(\theta) d \theta+\sum_{j=1}^{\ell} A_{j} \Phi_{n}^{\ell+1}\left(z_{j}\right) \overline{\Phi_{n}^{\ell}\left(z_{j}\right)}=\frac{\gamma_{n}^{\ell+1}}{\gamma_{n}^{\ell}} .
$$

On the other hand, the orthonormality of $\Phi_{n}^{\ell+1}(z)$ yields (on writing $\Phi_{n}^{\ell}(z)=\left(\gamma_{n}^{\ell}\right)$ $\left.\gamma_{n}^{\ell+1}\right) \Phi_{n}^{\ell+1}(z)+q(z)$, with $\left.q \in \mathscr{P}_{n-1}\right)$

$$
\begin{aligned}
\frac{1}{2 \pi} \int_{-\pi}^{+\pi} & \Phi_{n}^{\ell+1}(z) \cdot \overline{\Phi_{n}^{\ell}(z)} \rho(\theta) d \theta+\sum_{j=1}^{\ell} A_{j} \Phi_{n}^{\ell+1}\left(z_{j}\right) \overline{\Phi_{n}^{\ell}\left(z_{j}\right)} \\
= & \frac{1}{2 \pi} \int_{-\pi}^{+\pi} \Phi_{n}^{\ell+1}(z) \cdot \overline{\Phi_{n}^{\ell}(z)} \rho(\theta) d \theta+\sum_{j=1}^{\ell+1} A_{j} \Phi_{n}^{\ell+1}\left(z_{j}\right) \overline{\Phi_{n}^{\ell}\left(z_{j}\right)} \\
& -A_{\ell+1} \Phi_{n}^{\ell+1}\left(z_{\ell+1}\right) \overline{\Phi_{n}^{\ell}\left(z_{\ell+1}\right)} \\
= & \frac{\gamma_{n}^{\ell}}{\gamma_{n}^{\ell+1}}-A_{\ell+1} \Phi_{n}^{\ell+1}\left(z_{\ell+1}\right) \overline{\Phi_{n}^{\ell}\left(z_{\ell+1}\right)} .
\end{aligned}
$$

So we have

$$
\frac{\gamma_{n}^{\ell}}{\gamma_{n}^{\ell+1}}=\frac{\gamma_{n}^{\ell+1}}{\gamma_{n}^{\ell}}+A_{\ell+1} \Phi_{n}^{\ell+1}\left(z_{\ell+1}\right) \overline{\Phi_{n}^{\ell}\left(z_{\ell+1}\right)}
$$

and finally

$$
\left(\frac{k_{n}}{\gamma_{n}^{\ell+1}}\right)^{2}=\left(\frac{k_{n}}{\gamma_{n}^{\ell}}\right)^{2}\left[1+A_{\ell+1} \frac{\gamma_{n}^{\ell}}{\gamma_{n}^{\ell+1}} \Phi_{n}^{\ell+1}\left(z_{\ell+1}\right) \cdot \overline{\Phi_{n}^{\ell}\left(z_{\ell+1}\right)}\right]
$$

As in the proof of [8, Corollary 5], by the reproducing property of the kernel $R_{n}^{l}(z, \xi)$, and using a similar Christoffel-Darboux formula (we consider here $\Phi_{n}^{\ell+1}, \Phi_{n}^{\ell}$, and $R_{n}^{l}(z, \xi)$ instead of $\Phi_{n}, \varphi_{n}$, and $K_{n}(z, \xi)$ in [8], respectively), we get that

$$
\Phi_{n}^{\ell+1}(z)-\left(\frac{\gamma_{n}^{\ell+1}}{\gamma_{n}^{\ell}}\right) \Phi_{n}^{\ell}(z)=-A_{\ell+1} \Phi_{n}^{\ell+1}\left(z_{\ell+1}\right) \cdot R_{n}^{l}\left(z_{\ell+1}, z\right) .
$$


48 Asymptotics for orthogonal polynomials off the circle

Letting $z=z_{\ell+1}$ in (5.28) gives

$$
\left(\frac{\gamma_{n}^{\ell}}{\gamma_{n}^{\ell+1}}\right) \Phi_{n}^{\ell+1}\left(z_{\ell+1}\right)=\frac{\Phi_{n}^{\ell}\left(z_{\ell+1}\right)}{1+A_{\ell+1} R_{n}^{l}\left(z_{\ell+1}, z_{\ell+1}\right)} .
$$

Thus, (5.27) and (5.29) imply that

$$
\begin{aligned}
\left(\frac{k_{n}}{\gamma_{n}^{\ell+1}}\right)^{2} & =\left(\frac{k_{n}}{\gamma_{n}^{\ell}}\right)^{2}\left[1+\frac{\left|\Phi_{n}^{\ell}\left(z_{\ell+1}\right)\right|^{2}}{1 /\left(A_{\ell+1}\right)+R_{n}^{l}\left(z_{\ell+1}, z_{\ell+1}\right)}\right] \\
& =\left(\frac{k_{n}}{\gamma_{n}^{\ell}}\right)^{2}\left[1+\left(\left|z_{\ell+1}\right|^{2}-1\right)\left(\frac{\left(\left|z_{\ell+1}\right|^{2}-1\right)}{A_{\ell+1}\left|\Phi_{n}^{\ell}\left(z_{\ell+1}\right)\right|^{2}}+1-\left|\frac{\left(\Phi_{n}^{\ell}\right)^{*}\left(z_{\ell+1}\right)}{\Phi_{n}^{\ell}\left(z_{\ell+1}\right)}\right|^{2}\right)^{-1}\right] .
\end{aligned}
$$

This, with (3.3), implies that

$$
\left(\frac{k_{n}}{\gamma_{n}^{\ell+1}}\right)^{2} \leq\left(\prod_{j=1}^{\ell}\left|z_{j}\right|^{2}\right)\left(1+\left|z_{\ell+1}\right|^{2}-1\right)=\prod_{j=1}^{\ell+1}\left|z_{j}\right|^{2} .
$$

Theorem 5.3. Consider a measure $v$ of the form

$$
\nu=\frac{\mu}{2 \pi}+\sum_{k=1}^{\infty} A_{k} \delta\left(z-z_{k}\right)
$$

such that $v \in A$. Then

$$
\lim _{n \rightarrow \infty} \mu_{n}(\nu)=\mu(\nu)
$$

Proof

(A) An upper bound of limsup $\mu_{n}(\nu)$. If $\nu \in A$, then

$$
\left(\frac{k_{n}}{\gamma_{n}^{\ell}}\right)^{2} \leq\left(\prod_{k=1}^{\ell}\left|z_{k}\right|\right)^{2} \quad \forall n, \forall \ell .
$$

Since

$$
\mu_{n}(\ell)=\frac{1}{\left(\gamma_{n}^{\ell}\right)^{2}}, \quad \mu_{n}(\rho)=\frac{1}{\left(k_{n}\right)^{2}}
$$

we have that

$$
\mu_{n}(\ell) \leq \mu_{n}(\rho)\left[\prod_{k=1}^{\ell}\left|z_{k}\right|\right]^{2}
$$


Hence, taking limit when $\ell$ tends to infinity and using Theorem 5.1,

$$
\mu_{n}(\nu) \leq \mu_{n}(\rho)\left[\prod_{k=1}^{\infty}\left|z_{k}\right|\right]^{2}
$$

On the other hand, it is well known that (see $[3,7,12])$

$$
\lim _{n \rightarrow \infty} \frac{1}{\left(k_{n}\right)^{2}}=\lim _{n \rightarrow \infty} \mu_{n}(\rho)=\lim _{n \rightarrow \infty} m_{n}(\rho)=\mu(\rho)=(D(\infty))^{2},
$$

and by Lemma 4.4,

$$
\limsup _{n \rightarrow \infty} \mu_{n}(\nu) \leq \mu(\rho)\left[\prod_{k=1}^{\infty}\left|z_{k}\right|\right]^{2}=\mu(\nu) .
$$

(B) A lower bound of $\liminf _{n \rightarrow \infty} \mu_{n}(\nu)$. We will present two proofs of this affirmation.

(B1) First proof. For extremal functions $\psi_{n}^{\infty}$, the relation (5.39) leads to

$$
\left\|\psi_{n}^{\infty}\right\|_{\rho}^{2} \leq M=\text { constant }
$$

Inequality (5.40) and Theorem 2.1 imply that

$$
\sup _{z \in K}\left|\psi_{n}^{\infty}(z)\right| \leq C(K) M=\text { constant. }
$$

For every compact set $K \subset G$, put

$$
V=\liminf _{n \rightarrow \infty}\left\|\psi_{n}^{\infty}\right\|_{\rho}^{2}
$$

then for some subsequence $\left\{\left\|\psi_{n}^{\infty}\right\|_{\rho}\right\}_{n \in \Lambda}, V=\lim _{(n \in \Lambda)}\left\|\psi_{n}^{\infty}\right\|_{\rho}^{2}<+\infty$. From (5.41), we deduce that $\left\{\psi_{n}^{\infty}\right\}_{n \in \Lambda}$ is a normal (or Montel) family in $G$. Therefore, we can find a function $\varphi$ that is the uniform limit (on the compact subsets of $G$ ) of some subsequence $\left\{\psi_{n}^{\infty}\right\}_{n \in \Lambda_{1}}$ of $\left\{\psi_{n}^{\infty}\right\}_{n \in \Lambda}(\operatorname{see}[10])$. From Lemma 2.2, we get that

$$
\|\varphi\|_{\rho}^{2} \leq \liminf _{n \rightarrow \infty\left(n \in \Lambda_{1}\right)}\left\|\psi_{n}^{\infty}\right\|_{\rho}^{2}=\liminf _{n \rightarrow \infty}\left\|\psi_{n}^{\infty}\right\|_{\rho}^{2}, \quad \varphi \in H^{2}(G, \rho) .
$$

Inequality (5.43) with the definition of $\mu_{n}(\nu)$ imply that

$$
\|\varphi\|_{\rho}^{2} \leq \liminf _{n \rightarrow \infty} \mu_{n}(\nu) .
$$

On the other hand, it is obvious that $\varphi(\infty)=1$, and (5.39) implies that

$$
\sum_{k=1}^{\infty} A_{k}\left|\Phi\left(z_{k}\right)\right|^{2 n}\left|\psi_{n}^{\infty}\left(z_{k}\right)\right|^{2} \leq M=\text { constant. }
$$


Since $\left|z_{k}\right|>1$, then $\lim _{n \rightarrow \infty} \psi_{n}^{\infty}\left(z_{k}\right)=0$, so we have $\varphi\left(z_{k}\right)=0, k \geq 1$. Finally, from (5.44), we get that

$$
\mu(\nu) \leq\|\varphi\|_{\rho}^{2} \leq \liminf _{n \rightarrow \infty} \mu_{n}(\nu) .
$$

(B2) Second proof. Using (5.5), we have that

$$
\mu_{n}(\nu) \geq \mu_{n}(\ell) \quad \forall \ell>0 .
$$

It is well known [5, pages 40-43] that

$$
\lim _{n \rightarrow \infty} \mu_{n}(\ell)=\mu(\ell)
$$

where

$$
\begin{gathered}
\mu(\ell)=\inf \left\{\frac{1}{2 \pi} \int_{-\pi}^{+\pi}\left|\psi\left(e^{i \theta}\right)\right|^{2} \rho(\theta) d \theta: \psi \in H^{2}(G, \rho), \psi(\infty)=1,\right. \\
\left.\psi\left(z_{k}\right)=0, k=1,2, \ldots, \ell\right\} .
\end{gathered}
$$

We also have (see [5, Lemma 4.2])

$$
\mu(\ell)=\mu(\rho)\left[\prod_{k=1}^{\ell}\left|z_{k}\right|\right]^{2} .
$$

We then obtain that

$$
\liminf _{n \rightarrow \infty} \mu_{n}(\nu) \geq \mu(\rho)\left[\prod_{k=1}^{\ell}\left|z_{k}\right|\right]^{2} \quad \forall \ell>0 .
$$

Finally, by using (4.21), we obtain that

$$
\liminf _{n \rightarrow \infty} \mu_{n}(\nu) \geq \mu(\rho)\left[\prod_{k=1}^{\infty}\left|z_{k}\right|\right]^{2}=\mu(\nu) .
$$

Next, we state the asymptotic formula of the orthonormal polynomials $\left\{\Phi_{n}(z)\right\}$

Theorem 5.4. Consider a measure $v$ of the form

$$
v=\frac{\mu}{2 \pi}+\sum_{k=1}^{\infty} A_{k} \delta\left(z-z_{k}\right)
$$

such that $v \in A$, and $\left\{\Phi_{n}(z)\right\}_{n=1}^{\infty}$ is the system of orthonormal polynomials associated to $v$ which is satisfying relations (1.4). Then

$$
\begin{gathered}
\lim _{n \rightarrow \infty}\left\|\psi_{n}^{\infty}-\psi^{\infty}\right\|_{\rho}^{2}=0 \\
\lim _{n \rightarrow \infty} \frac{\Phi_{n}(z)}{z^{n}}=\left(\prod_{k=1}^{\infty} \frac{z-z_{k}}{\left(z \cdot \overline{z_{k}}\right)-1} \cdot \frac{\left|z_{k}\right|}{z_{k}}\right) \frac{1}{\overline{D(\bar{z})}},
\end{gathered}
$$


uniformly for every compact subset of $\{z \in \mathbb{C}:|z|>1\} . \psi_{n}^{\infty}$ and $\psi^{\infty}$ are, respectively, the optimal solutions of the extremal problems (4.3) and (4.7).

Proof. Set

$$
H_{n}=\frac{1}{2}\left(\psi_{n}^{\infty}+\psi^{\infty}\right)
$$

then

$$
H_{n}(\infty)=1, \quad \lim _{n \rightarrow \infty} H_{n}\left(z_{k}\right)=0, \quad k=1,2, \ldots
$$

This implies that

$$
\liminf _{n \rightarrow \infty}\left\|H_{n}\right\|_{\rho}^{2} \geq \mu(\nu)
$$

Using the parallelogram law in $H^{2}(G, \rho)$, we obtain that

$$
\left\|\psi_{n}^{\infty}-\psi^{\infty}\right\|_{\rho}^{2}=2\left(\left\|\psi_{n}^{\infty}\right\|_{\rho}^{2}+\left\|\psi^{\infty}\right\|_{\rho}^{2}\right)-4\left\|H_{n}\right\|_{\rho}^{2} .
$$

Hence

$$
\limsup _{n \rightarrow \infty}\left\|\psi_{n}^{\infty}-\psi^{\infty}\right\|_{\rho}^{2} \leq 2(\mu(\nu)+\mu(\nu))-4 \mu(\nu)=0
$$

so that

$$
\lim _{n \rightarrow \infty}\left\|\psi_{n}^{\infty}-\psi^{\infty}\right\|_{\rho}^{2}=0
$$

There remains the asymptotic formula (5.55). Recall that

$$
\begin{gathered}
\psi_{n}^{\infty}(z)=\frac{1}{\gamma_{n}} \frac{\Phi_{n}(z)}{z^{n}} \\
\psi^{\infty}(z)=\hat{\psi}(z) \cdot B_{\infty}(z)=\hat{\psi}(z) \cdot \prod_{k=1}^{\infty} \frac{z-z_{k}}{z \overline{z_{k}}-1} \frac{\left|z_{k}\right|^{2}}{z_{k}} .
\end{gathered}
$$

It is not difficult to show that

$$
\hat{\psi}(z)=\frac{D(\infty)}{\overline{D(\bar{z})}}
$$

Let $K \subset\{z \in \mathbb{C}:|z|>1\}$ be a compact set. Theorem 2.1 implies that

$$
\lim _{n \rightarrow \infty}\left\{\operatorname{Max}\left\{\left|\frac{1}{\gamma_{n}} \frac{\Phi_{n}(z)}{z^{n}}-\frac{D(\infty)}{\overline{D(\bar{z})}} \cdot \prod_{k=1}^{\infty} \frac{z-z_{k}}{z \overline{z_{k}}-1} \frac{\left|z_{k}\right|^{2}}{z_{k}}\right|: z \in K\right\}\right\}=0
$$


On the other hand, we know that (see Theorem 5.3, Lemma 4.4, and [12])

$$
\begin{gathered}
\left(\frac{1}{\gamma_{n}}\right)^{2}=\mu_{n}(\nu), \quad \lim _{n \rightarrow \infty} \mu_{n}(\nu)=\mu(\nu)=\mu(\rho) \cdot\left(\prod_{k=1}^{\infty}\left|z_{k}\right|\right)^{2}, \\
\mu(\rho)=(D(\infty))^{2} .
\end{gathered}
$$

We obtain

$$
\lim _{n \rightarrow \infty} \frac{1}{\gamma_{n}}=\left(\prod_{k=1}^{\infty}\left|z_{k}\right|\right)(D(\infty))
$$

To get the asymptotic formula (5.55), we use (5.64) and (5.66) and the fact that

$$
\left(\prod_{k=1}^{\infty} \frac{z-z_{k}}{z \overline{z_{k}}-1} \frac{\left|z_{k}\right|^{2}}{z_{k}}\right)=\left(\prod_{k=1}^{\infty}\left|z_{k}\right|\right)\left(\prod_{k=1}^{\infty} \frac{z-z_{k}}{\left(z \cdot \overline{z_{k}}\right)-1} \cdot \frac{\left|z_{k}\right|}{z_{k}}\right) .
$$

\section{References}

[1] N. I. Akhiezer and M. G. Krein, Some Problems of the Theory of Moments, Gos. Naucno-Tehn. Izdat. Ukarine, 1938 (Russian), English version in Translations of Mathematical Monographs, vol. 2, American Mathematical Society, Rhode Island, 1962.

[2] R. Benzine, Asymptotic behavior of orthogonal polynomials corresponding to a measure with infinite discrete part off a curve, J. Approx. Theory 89 (1997), no. 2, 257-265.

[3] Ya. L. Geronimus, On some extremal problems in the space $L_{\sigma}^{(p)}$, Mat. Sb. (N.S.) 31(73) (1952), 3-26 (Russian).

[4] , Polynomials Orthogonal on a Circle and Interval, International Series of Monographs on Pure and Applied Mathematics, vol. 18, Pergamon Press, New York, 1960.

[5] V. Kaliaguine and R. Benzine, Sur la formule asymptotique des polynômes orthogonaux associés à une mesure concentrée sur un contour plus une partie discrète finie [An asymptotic formula for orthogonal polynomials associated with a measure concentrated on a contour plus a finite discrete part], Bull. Soc. Math. Belg. Sér. B 41 (1989), no. 1, 29-46 (French).

[6] R. Khaldi and R. Benzine, On a generalization of an asymptotic formula of orthogonal polynomials, Int. J. Appl. Math. 4 (2000), no. 3, 261-274.

[7] - Asymptotic behavior of orthogonal polynomials corresponding to a measure with infinite discrete part off an arc, Int. J. Math. Math. Sci. 26 (2001), no. 8, 449-455.

[8] P. Koosis, Introduction to $H_{p}$ Spaces, London Mathematical Society Lecture Note Series, vol. 40, Cambridge University Press, Cambridge, 1980.

[9] X. Li and K. Pan, Asymptotic behavior of orthogonal polynomials corresponding to measure with discrete part off the unit circle, J. Approx. Theory 79 (1994), no. 1, 54-71.

[10] W. Rudin, Real and Complex Analysis, McGraw-Hill, New York, 1968.

[11] V. I. Smirnov and N. A. Lebedev, The Constructive Theory of Functions of a Complex Variable, Izdat. Nauka, Moscow, 1964 (Russian), English translation: MIT Press, Massachusetts, 1968.

[12] V. J. Smirnov, Sur la théorie des polynômes orthogonaux à une variable complexe, Journal de la Société Physico-Mathématique de Leningrad 2 (1928), 155-179 (French).

[13]_- Sur les formules de Cauchy et de Green et quelques problèmes qui s'y rattachent, Bulletin de l'Académie des Sciences de l'URSS (1932), 337-372 (French). 
[14] G. Szegö, Orthogonal Polynomials, 4th ed., American Mathematical Society, Colloquium Publications, vol. 23, American Mathematical Society, Rhode Island, 1975.

R. Khaldi: Department of Mathematics, University of Annaba, BP 12, 2300 Annaba, Algeria E-mail address: rkhadi@yahoo.fr

R. Benzine: Department of Mathematics, University of Annaba, BP 12, 2300 Annaba, Algeria E-mail address: benzine@ifrance.com 


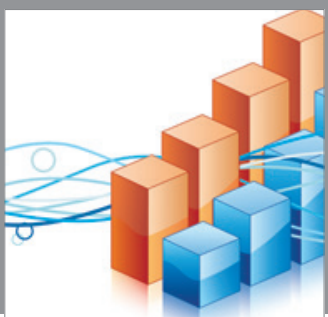

Advances in

Operations Research

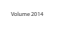

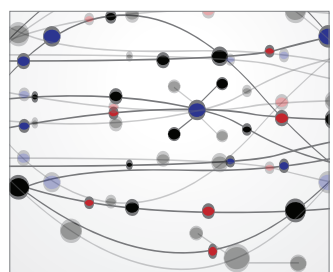

\section{The Scientific} World Journal
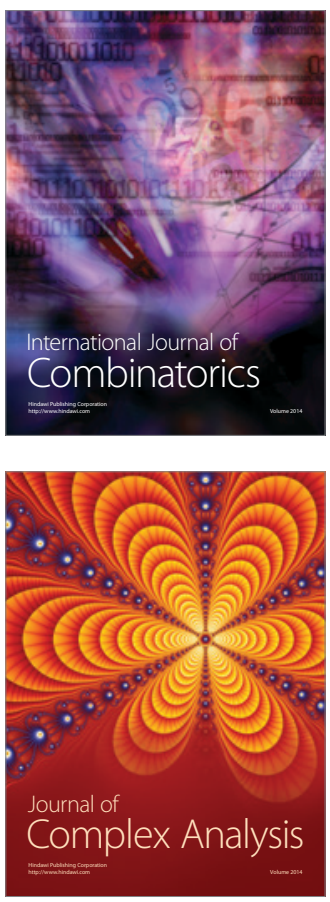

International Journal of

Mathematics and

Mathematical

Sciences
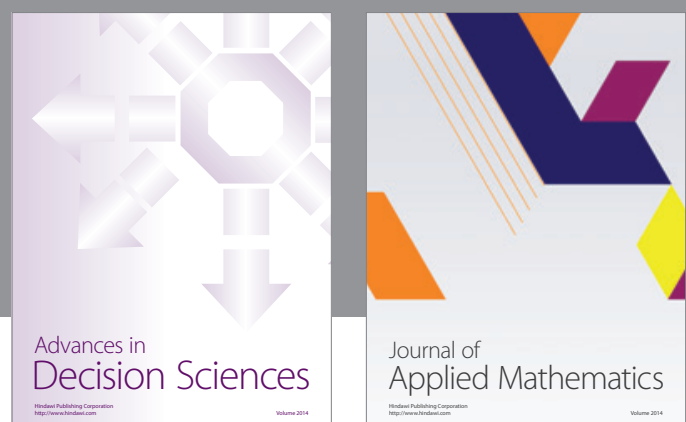

Journal of

Applied Mathematics
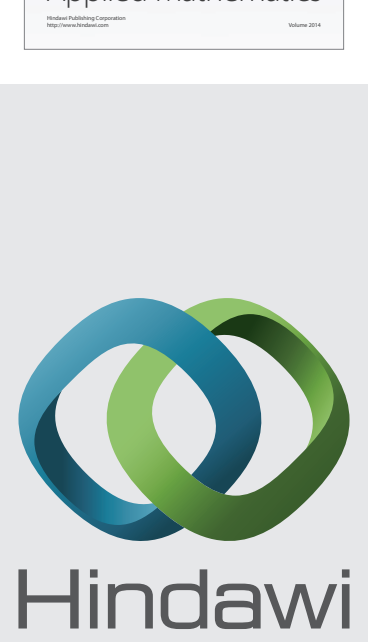

Submit your manuscripts at http://www.hindawi.com
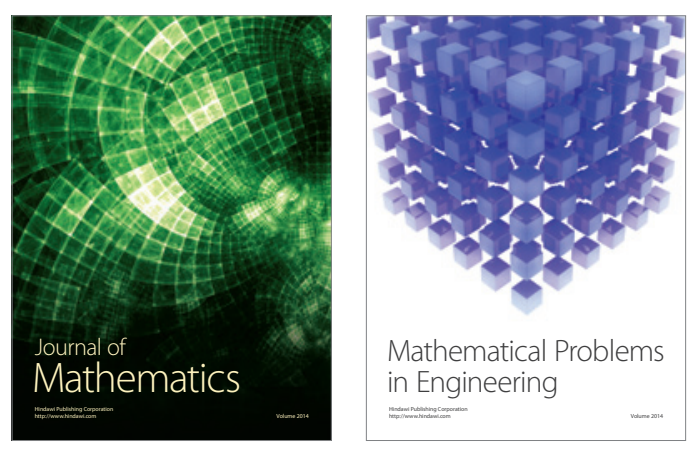

Mathematical Problems in Engineering
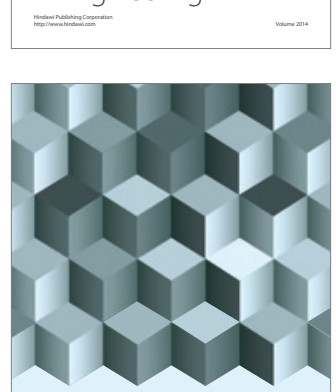

Journal of

Function Spaces
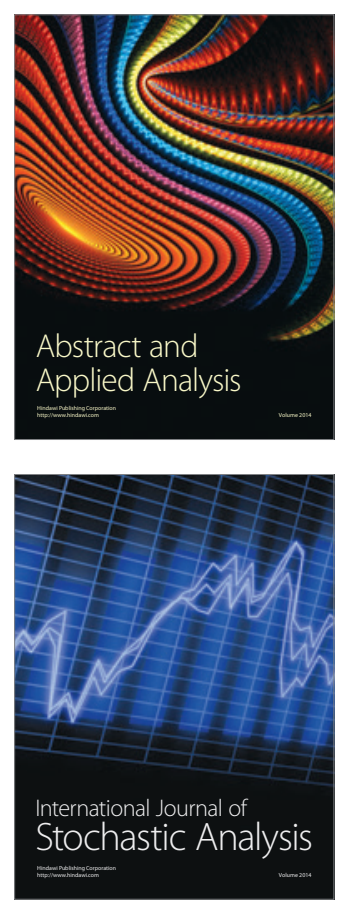

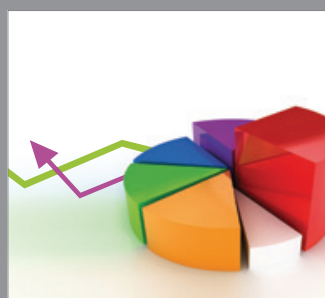

ournal of

Probability and Statistics

Promensencen
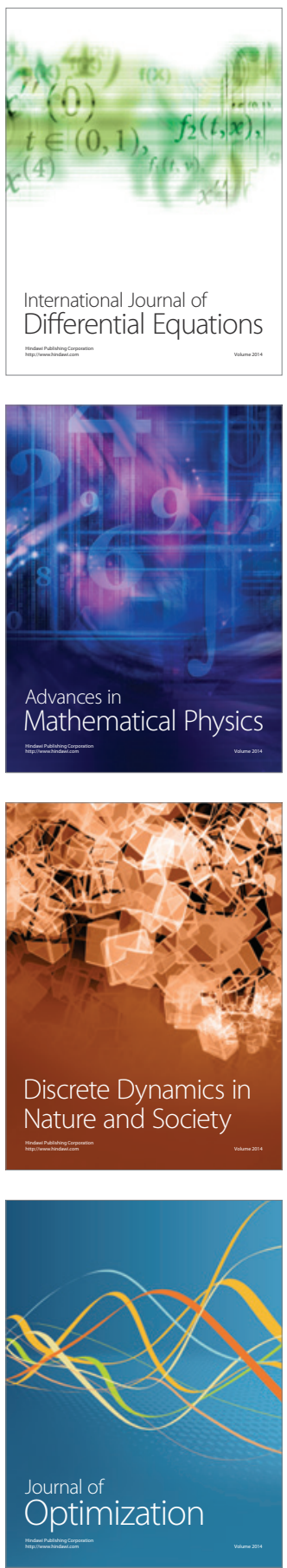